\title{
REDUCIBILITY FOR REAL ANALYTIC QUASI-PERIODIC LINEAR SYSTEMS
}

\author{
G. C. O'BRIEN
}

(Received 18 September 1974)

\begin{abstract}
In this paper we examine the linear differential equations $x^{\prime}=A x+P(\phi) x, \phi^{\prime}=\omega$ where $x \in R^{n}, \phi \in R^{m}, A$ and $\omega$ are constant and $P(\phi)$ is real analytic and periodic in $\phi$. We use the method of accelerated convergence to overcome the small divisors problem and reduce this system to the system $y^{\prime}=B y, \phi^{\prime}=\omega$ with constant coefficients.

This problem has already been examined by Mitropolśkiĭ and Samoilenko but the calculations and details in their work are formidable and difficult to follow. Besides being simpler our method provides more precise estimates at all stages and can be extended to the differentiable case to provide a significant improvement over previous results.
\end{abstract}

1.

In this paper we are concerned with the linear differential equations

$$
\begin{aligned}
& x^{\prime}=A x+P(\phi) x \\
& \phi^{\prime}=\omega
\end{aligned}
$$

where $x \in R^{n}, \phi \in R^{m}, A$ is a constant $n \times n$ matrix, $\omega$ is a constant vector in $R^{m}$ and the $n \times n$ matrix $P(\phi)$ is holomorphic and periodic in $\phi_{i}$ with period $2 \pi$ for $i=1, \cdots, m$. Thus we are considering a quasi-periodic system.

Provided $|P(\phi)|$ is sufficiently small we obtain a quasi-periodic transformation $x=[I+U(w t)] y$, where $|U(w t)|$ is small, which transforms (1) into the system with constants coefficients

$$
\begin{aligned}
y^{\prime} & =B y \\
\phi^{\prime} & =\omega
\end{aligned}
$$

where $|A-B|$ is also small. This entails a sequence of such transformations and our proof uses the method of accelerated convergence to overcome the celebrated "small divisors" difficulty.

This problem has already been examined by Mitropolśkiı and Samoilenko (1969) and (1965) but the calculations and details in their work are formidable and difficult to follow. Besides being simpler, our method provides more precise estimates at all stages and corrects a technical difficulty in their proofs. 
Moreover our technique can be extended to the case where $P(\phi)$ is $l$ times differentiable to obtain a significant improvement over any previous results. Our estimates have recently been used by Gray (to appear) to illustrate an application of his implicit function theorem for small divisor problems.

2.

In this course of finding such a reduction of the given system of differential equations we will be led to consider, at each step, matrix equations of the form

$$
\left(\frac{\partial U}{\partial \phi}, \omega\right)+U A-A U=C(\phi)
$$

where $\left(\frac{\partial U}{\partial \phi}, \omega\right)$ represents $\sum_{\alpha=1}^{m} \frac{\partial U}{\partial \phi_{\alpha}} \omega_{\alpha}$. We will do this by trying to find a solution with Fourier series

$$
U(\phi) \sim \sum_{|k|=0}^{\infty} U_{k} e^{i(k \cdot \phi)}
$$

and by equating coefficients we obtain equations of the form

$$
(i(k, \omega) I-A) U_{k}+U_{k} A=C_{k} .
$$

That is, we have the general linear matrix equation

$$
A_{1} X+X B_{1}=C_{1} \text {. }
$$

This equation has a unique solution if and only if $A_{1}$ and $B_{1}$ do not have any common eigenvalues.

We define the norm of a column vector $x=\left(x_{1} ; \cdots ; x_{m}\right)$ by

$$
|x|=\sum_{\alpha}\left|x_{\alpha}\right| \text {. }
$$

We define the norm of a row vector $k=\left(k_{1}, \cdots, k_{m}\right)$ by

$$
|k|=\max _{\alpha}\left|k_{\alpha}\right| .
$$

Then for the scalar product

$$
(k, x)=k_{1} x_{1}+\cdots+k_{m} x_{m}
$$

we have the obvious inequality

$$
|(k, x)| \leqq|k||x|
$$

Finally, we define the norm of a matrix $A=\left(a_{i j}\right)$ by 


$$
|A|=\max _{\beta} \sum_{\alpha}\left|a_{\alpha \beta}\right| \text {. }
$$

ve need the following preliminary result.

THEOREM 1.

Suppose

(i) $A=\operatorname{diag}\left(\lambda_{1}, \cdots, \lambda_{n}\right)$ is a real diagonal matrix with distinct eigenralues;

(ii) $P(\phi)$ is an $n \times n$ matrix function of the $m$-vector $\phi$ which is real for real $p$, has period $2 \pi$ in each coordinate $\phi_{\alpha}$ and which is holomorphic and satisfies he inequality

$$
|P(\phi)| \leqq M
$$

n the strip $|\operatorname{Im} \phi|<\rho$;

(iii) $\omega$ is a real $m$-vector such that for all integral $m$-vectors $k \neq 0$

$$
|(k, \omega)| \geqq \gamma|k|^{-\tau}
$$

where $\tau>m$ and $\gamma>0$.

Then the partial differential equation

$$
\left(\frac{\partial U}{\partial \phi}, \omega\right)+U A-A U=P(\phi)
$$

has a solution which is real for real $\phi$, has period $2 \pi$ in each coordinate $\phi_{\alpha}$ and which is holomorphic and satisfies the inequality

$$
\left|U(\phi)-U_{0}\right| \leqq c \gamma^{-1} \delta^{-\tau} M,
$$

in the strip $|\operatorname{Im} \phi| \leqq \rho-\delta(\delta<1)$, where

$$
U_{0}=\frac{1}{(2 \pi)^{m}} \int_{0}^{2 \pi} \cdots \int_{0}^{2 \pi} U(\phi) d \phi_{1} \cdots d \phi_{m}
$$

is the mean value of $U(\phi)$ and $c=c(m, \tau)>0$.

Proof. Let

$$
P(\phi)=\sum_{k} P_{k} e^{i(k, \phi)}
$$

be the Fourier expansion of $P(\phi)$, so that

$$
P_{k}=\frac{1}{(2 \pi)^{m}} \int_{0}^{2 \pi} \cdots \int_{0}^{2 \pi} P(\phi) e^{-i(k, \phi)} d \phi_{1} \cdots d \phi_{m} .
$$

By shifting the lines of integration to $\operatorname{Im} \phi_{\alpha}= \pm \rho_{\alpha}$, where $\sum_{\alpha} \rho_{\alpha} \leqq \rho$, we obtain

$$
\left|P_{k}\right| \leqq M e^{-\rho|k|}
$$


We look for a solution of (2) with the Fourier expansion

$$
U(\phi)=\sum_{k} U_{k} e^{i(k, \phi)}
$$

Substituting in (2) and equating coefficients we obtain

$$
U_{k}(A+i(k, \omega))-A U_{k}=P_{k} .
$$

Thus if we write $P_{k}=\left(p_{\alpha \beta}^{(k)}\right), U_{k}=\left(u_{\alpha \beta}^{(k)}\right)$, then

$$
\boldsymbol{u}_{\alpha \beta}^{(k)}=\frac{p_{\alpha \beta}^{(k)}}{\lambda_{\beta}-\lambda_{\alpha}+i(k, \omega)}, \alpha, \beta=1, \cdots, n .
$$

Since $P_{-k}=\bar{P}_{k}$ it follows that $U_{-k}=\bar{U}_{k}$. Also for $k \neq 0$

$$
\left|u_{\alpha \beta}^{(k)}\right| \leqq\left|p_{\alpha \beta}^{(k)}\right| /|(k, \omega)|, \alpha, \beta=1, \cdots, n,
$$

and hence

$$
\left|U_{k}\right| \leqq\left|P_{k}\right| /|(k, \omega)| .
$$

It. follows that for $|\operatorname{Im} \phi| \leqq \rho-\delta$

$$
\left|U(\phi)-U_{0}\right| \leqq M \sum_{k \neq 0} \frac{1}{|(k, \omega)|} e^{-\delta|k|} .
$$

It remains to establish the convergence of the series on the right and obtain a sharp estimate for its sum.

Let $K_{j}$ denote the set of all integral vectors $k$ such that

$$
\begin{array}{ll}
2^{-j-2}<|(k, \omega)| \leqq 2^{-j-1} & (j=0,1, \cdots) \\
2^{-1}<|(k, \omega)| & (j=-1) .
\end{array}
$$

Every non-zero integral vector belongs to one and only one set $K_{j}$. Hence

$$
\begin{aligned}
S & \equiv \sum_{k \neq 0} \frac{1}{|(k, \omega)|} e^{-\delta|k|}=\sum_{j=0}^{\infty} \sum_{k \in K_{i}} \frac{1}{|(k, \omega)|} e^{-\delta|k|} \\
& +\sum_{|(k, \omega)|>\frac{1}{2}} \frac{1}{|(k, \omega)|} e^{-\delta|k|} \\
& \leqq \sum_{j=-1}^{\infty} 2^{i+2} \sum_{k \in K_{j}} e^{-\delta|k|}
\end{aligned}
$$

For $k \in K_{j}$ and $j=0,1, \cdots$

$$
\gamma|k|^{-\tau} \leqq|(k, \omega)| \leqq 2^{-i-1}<2^{-i}
$$

and hence

$$
|k| \geqq \alpha_{j}=\left(2^{j} \gamma\right)^{1 / \tau}
$$


For distinct $k_{1}, k_{2} \in K_{i}$

$$
\gamma\left|k_{1}-k_{2}\right|^{-\tau} \leqq\left|\left(k_{1}-k_{2}, \omega\right)\right| \leqq\left|\left(k_{1}, \omega\right)\right|+\left|\left(k_{2}, \omega\right)\right| \leqq 2^{-j}
$$

hence $\left|k_{1}-k_{2}\right| \geqq \alpha_{i}$.

For any $k \in K_{j}$ let $\mathscr{W}_{k}$ denote the open cube with centre $k$ and sides parallel to the axes of length $\alpha_{j}$. The cubes are disjoint since $y \in \mathscr{W}_{k_{1}} \cap \mathscr{W}_{k_{2}}$ implies

$$
a_{i} \leqq\left|k_{1}-k_{2}\right| \leqq\left|y-k_{1}\right|+\left|y-k_{2}\right|<\frac{1}{2} a_{j}+\frac{1}{2} a_{j}=a_{j} .
$$

Let $\nu_{i l}$ denote the number of $k \in K_{i}$ such that

$$
l a_{j} \leqq|k|<(l+1) a_{j} \quad(l=1,2, \cdots) .
$$

The corresponding cubes $\mathscr{W}_{k}$ lie in the set $\left(l-\frac{1}{2}\right) a_{j}<|y|<\left(l+\frac{3}{2}\right) a_{j}$ which has volume $\left[(2 l+3)^{m}-(2 l-1)^{m}\right] \alpha_{i}^{m}$. Since each cube has volume $a_{i}^{m}$ this gives

$$
\nu_{j l} \leqq(2 l+3)^{m}-(2 l-1)^{m} \leqq 4 m(2 l+3)^{m-1}
$$

by the mean value theorem. Since $2+{ }_{1}^{3} \leqq 5$ it follows that $\nu_{j l} \leqq 5^{m} m l^{m-1}$. Therefore

$$
\sum_{k \in K_{i}} e^{-\delta|k|} \leqq \sum_{i=1}^{\infty} \nu_{j l} e^{-\delta a_{i}} \leqq 5^{m} m \sum_{i=1}^{\infty} l^{m-1} e^{-\delta l a_{i}}
$$

Put $q=e^{-\delta a_{i}}$. Since $t^{m-1} q^{t / 2}$ takes its maximum value for $\frac{1}{2} t \log \frac{1}{q}=m-1$ we have

and hence

$$
\begin{aligned}
\sum_{i=1}^{\infty} l^{m-1} q^{\prime} & =\sum_{i=1}^{\infty}\left(l^{m-1} q^{1 / 2}\right) q^{1 / 2} \\
& \leqq\left(\frac{m}{\log 1 / q}\right)^{m-1} \sum_{i=1}^{\infty} q^{1 / 2}=\left(\frac{m}{\log 1 / q}\right)^{m-1}=\left(q^{-\frac{1}{2}}-1\right)^{-1}
\end{aligned}
$$

$$
\sum_{k \in K_{i}} e^{-\delta|k|} \leqq(5 m)^{m}\left(\delta a_{j}\right)^{1-m}\left(e^{\delta a / 2}-1\right)^{-1} .
$$

The number of $k$ with $|k|=r$ is

$$
n_{k} \leqq(2 r+1)^{m}-(2 r-1)^{m} \leqq 4 m(2 r+1)^{m-1} \leqq 4.3^{m-1} m r^{m-1} .
$$

Therefore, when $j=-1$,

$$
S_{-1}=2 \sum_{\mid k, \omega) \mid>2} e^{-\delta|k|} \leqq 8 m \cdot 3^{m-1} \sum_{r=1}^{\infty} r^{m-1} e^{-8 r} \leqq 8 m 3^{m-1}\left(\frac{m}{\delta}\right)^{m-1}\left(e^{\frac{1}{2} \delta}-1\right)^{-1} .
$$

It follows from the definition of $a_{i}$ that

$$
S-S_{-1} \leqq(5 m)^{m} \sum_{i=0}^{\infty} 2^{j+2}\left(\delta a_{j}\right)^{1-m}\left(e^{\delta a_{j} / 2}-1\right)^{-1}=(5 m)^{m} \sum_{j=0}^{\infty} 2^{j+2} g\left(2^{i+1}\right)
$$


where

$$
g(t)=\left[\delta(\gamma t / 2)^{1 / \tau}\right]^{1-m}\left[e^{\frac{1}{2} \delta(\gamma t / 2)^{1 / \tau}}-1\right]^{-1} .
$$

Since $2^{j+2}=4\left(2^{j+1}-2^{j}\right)$ and $g$ is a decreasing function this implies

$$
\begin{aligned}
S-S_{-1} & \leqq 4(5 m)^{m} \int_{1}^{\infty} g(t) d t \\
& \leqq 8(5 m)^{m} \tau \gamma^{-1} \delta^{-\tau} \int_{0}^{\infty} g\left(2 \gamma^{-1}\left(\frac{v}{\delta}\right)^{\tau}\right) v^{\tau-1} d v \\
& =8(5 m)^{m} \tau^{\gamma-1} \delta^{-\tau} \int_{0}^{\infty} v^{\tau-m}\left(e^{v / 2}-1\right)^{-1} d v \\
& =c_{1} \gamma^{-1} \delta^{-\tau}
\end{aligned}
$$

since $\tau>m$.

Similarly

$$
S_{-1} \leqq \frac{16}{3}\left(\frac{3 m}{2}\right)^{m}\left(\frac{\delta}{2}\right)^{1-m}\left(e^{\delta / 2}-1\right)^{-1} \leqq c_{2} \delta^{-m}
$$

Thus, for $\delta<1$ it follows that $S \leqq c \gamma^{-1} \delta^{-\tau}$. This proves that $U(\phi)$ is holomorphic and establishes the estimate for $U(\phi)-U_{0}$.

3.

Consider now the system of ordinary differential equations

$$
\begin{aligned}
x^{\prime} & =A x+P(\phi) x \\
\phi^{\prime} & =\omega
\end{aligned}
$$

where $A, P(\phi)$ and $\omega$ satisfy the hypothesis of Theorem 1. Thus

$$
\min _{\alpha \neq \beta}\left|\lambda_{\alpha}-\lambda_{\beta}\right| \geqq r>0 \text {. }
$$

Let $D(\phi)$ be the diagonal matrix with the same diagonal elements as $P(\phi)$ and let $D_{0}$ be its mean value. As in Theorem 1. we can find a matrix $U(\phi)$ such that

$$
\left(\frac{\partial U}{\partial \phi}, \omega\right)+U A-A U=P(\phi)-D_{0}
$$

Its mean value $U_{0}$ must satisfy

$$
U_{0} A-A U_{0}=P_{0}-D_{0} .
$$

If

$$
P_{0}=\left(p_{\alpha \beta}^{0}\right), \quad U_{0}=\left(u_{\alpha \beta}^{0}\right)
$$


this equation has the solution

$$
u_{\alpha \beta}^{0}=\left\{\begin{array}{lll}
\frac{p_{\alpha \beta}^{0}}{\lambda_{\beta}-\lambda_{\alpha}} & \text { for } & \alpha \neq \beta, \\
0 & \text { for } & \alpha=\beta .
\end{array}\right.
$$

Thus

$$
\left|U_{0}\right| \leqq \frac{1}{r}\left|P_{0}\right| \leqq \frac{M}{r}
$$

The remaining Fourier coefficients of $U(\phi)$ are unchanged and thus we obtain

$$
|U(\phi)| \leqq\left(r^{-1}+c \gamma^{-1} \delta^{-\tau}\right) M \quad \text { for }|\operatorname{Im} \phi| \leqq \rho-\delta .
$$

The change of variables $x=[I+U(\phi)] \tilde{x}$ transform (3) into a system

(4)

$$
\begin{aligned}
\tilde{x}^{\prime} & =\tilde{A} \tilde{x}+\tilde{P}(\phi) \tilde{x} \\
\phi^{\prime} & =\omega
\end{aligned}
$$

of the same form, where

$$
\tilde{A}=A+D_{0}, \tilde{P}=(I+U)^{-1}\left(P U-U D_{0}\right) .
$$

Thus if $|U| \leqq \frac{1}{2}$ then

$$
|\tilde{P}| \leqq\left|(I+U)^{-1}\right||U|\left(|P|+\left|D_{0}\right|\right) \leqq 4 M|U| .
$$

Moreover if $\alpha \neq \beta$

$$
\left|\tilde{\lambda_{\alpha}}-\tilde{\lambda_{\beta}}\right| \geqq\left|\lambda_{\sigma}-\lambda_{\beta}\right|-\left|p_{\alpha \alpha}^{0}-p_{\beta \beta}^{0}\right| \geqq r-2 M .
$$

Let $\varepsilon$ be any number such that $0<\epsilon<\min \left(1, \rho, \frac{1}{2} r\right)$. Choose $\theta(0<\theta<1)$ so small that if $\theta_{j}=\theta^{\left(\frac{3}{2}, j\right.}$ then

$$
\begin{aligned}
\sum_{j=0}^{\infty} \theta_{j}<\frac{1}{4} \varepsilon ; \sum_{j=0}^{\infty} \theta_{i}^{1 / \tau}<\frac{\epsilon}{a} \text { where } a=(8 c / \gamma)^{1 / \tau} \\
\qquad \sum_{j=0}^{\infty} \theta_{j}<\varepsilon / p \text { where } p=\prod_{j=0}^{\infty}\left(1+\frac{1}{4} \theta_{j}\right) .
\end{aligned}
$$

Put

$$
\rho_{i}=\rho-a\left(\theta_{0}^{1 / \tau}+\cdots+\theta_{j-1}^{1 / \tau}\right)>0, r_{i}=r-2\left(\theta_{0}+\cdots+\theta_{j-1}\right)>0,
$$

and suppose that the system (3) satisfies the above conditions with $M, \rho, r$ replaced by $\theta_{i}^{2}, \rho_{i}, r_{j}$. We will show that the transformed system (4) satisfies the same conditions with $M, \rho, r$ replaced by $\theta_{i+1}^{2}, \rho_{j+1}, r_{j+1}$. Thus we take $\delta=a \theta_{j}^{1 / \tau}$. By the definition of $a$ we have for $|\operatorname{Im} \phi| \leqq \rho_{i+1}$, 


$$
\left|U_{j+1}\right| \leqq\left(r_{j}^{-1}+\frac{1}{8} \theta_{j}^{-1}\right) \theta_{j}^{2} \leqq \frac{1}{4} \theta_{j},
$$

since $r_{j} \geqq 8 \theta_{j}$. Thus $\left|U_{j+1}\right| \leqq \frac{1}{2}$ and hence

$$
\left|P_{j+1}\right| \leqq 4 \theta_{j 4}^{2 \frac{1}{4}} \theta_{j}=\theta_{j}^{3}=\theta_{j+1}^{2} .
$$

Moreover

$$
\lim _{\alpha \neq \beta}\left|\tilde{\lambda_{\alpha}}-\tilde{\lambda_{\beta}}\right| \geqq r_{j}-2 \theta_{j}^{2} \geqq r_{j}-2 \theta_{j}=r_{j+1} .
$$

It follows that if in the original system (3), $M \leqq \theta^{2}$ then the above transformation can be repeated indefinitely. We will now prove the existence of a limit system. We have

where

$$
\begin{aligned}
& x_{j}^{\prime}=A_{j} x_{j}+P_{i}(\phi) x_{j} \\
& \phi^{\prime}=\omega
\end{aligned}
$$

$$
A_{i}=A+D_{0}^{(1)}+\cdots+D_{0}^{(j)} .
$$

Since $\left|P_{j}(\phi)\right| \leqq \theta_{j}^{2}$ for $\left.\mid \operatorname{Im} \phi\right) \leqq \rho_{j}$ it is obvious that as $j \rightarrow \infty, P_{j}(\phi) \rightarrow 0$ uniformly for $|\operatorname{Im} \phi|<\rho_{\infty}=\rho-a \sum_{s=0}^{\infty} \theta_{s}^{1 / \tau}$. Since $\left|D_{0}^{(j)}\right| \leqq \theta_{j}^{2}$ the existence of the limit, $B=\lim _{j \rightarrow \infty} A_{j}$ is also assured and $|B-A|<\varepsilon$. Finally we have $x=T_{i}(\phi) x_{j}$ where

$$
T_{j}=\left(I+U_{1}\right) \cdots\left(I+U_{j}\right) .
$$

Suppose that for some $j$

$$
\left|T_{j}\right| \leqq \prod_{s=0}^{j-1}\left(1+\frac{1}{4} \theta_{s}\right)
$$

With the convention $T_{0}=I$ this is certainly true for $j=0$. Since

$$
T_{i+1}=T_{i}\left(I+U_{j+1}\right)
$$

it follows that

$$
\left|T_{j+1}\right| \leqq\left|T_{j}\right|\left|\left(I+U_{i+1}\right)\right| \leqq \prod_{s=0}^{j}\left(1+\frac{1}{4} \theta_{s}\right) .
$$

Therefore for $|\operatorname{Im} \phi|<\rho_{\infty}$ we have $\left|T_{j}(\phi)\right| \leqq p$ for all $j$. Hence $\left|T_{i+1}-T_{j}\right| \leqq$ $\left|T_{j}\right|\left|U_{j+1}\right| \leqq \frac{1}{4} p \theta_{j}$

Thus the series $\Sigma\left\{T_{j+1}(\phi)-T_{i}(\phi)\right\}$ converges uniformly for $|\operatorname{Im} \phi|<\rho_{\infty}$. The sequence $\left\{T_{i}(\phi)\right\}$ converges uniformly in the same strip. Its limit $T(\phi)$ is holomorphic, is real for real $\phi$, and has period $2 \pi$ in each coordinate. Moreover, the partial derivatives $\partial T_{j} / \partial \phi_{\alpha}$ converge uniformly to $\partial T / \partial \phi_{\alpha}$. From

$$
T_{i+1}-I=T_{i}-I+T_{i} U_{i+1}
$$


we obtain

$$
|T-I| \leqq \sum_{j=0}^{\infty}\left|T_{j}\right|\left|U_{j+1}\right| \leqq \frac{1}{4} p \sum_{j=0}^{\infty} \theta_{j}<\varepsilon .
$$

Hence the matrix $T(\phi)$ is invertible and

$$
x_{j} \rightarrow y=T^{-1}(\phi) x \text { as } j \rightarrow \infty .
$$

Differentiating the relation $x=T_{j} x_{j}$ we obtain

$$
x^{\prime}=\left(\frac{\partial T_{j}}{\partial \phi}, \omega\right) x_{i}+T_{j} x_{j}^{\prime}
$$

Hence, letting $j \rightarrow \infty$

$$
x^{\prime}=\left(\frac{\partial T}{\partial \phi^{\prime}}, \omega\right) y+T B y .
$$

Differentiating the relation $x=T y$ we obtain

$$
x^{\prime}=\left(\frac{\partial T}{\partial \phi^{\prime}}, \omega\right) y+T y^{\prime} .
$$

Comparison of these two expressions for $x^{\prime}$ gives $y^{\prime}=B y$. Thus the change of variables $x=T(\phi) y$ transforms the system (3) into the autonomous system

$$
\begin{aligned}
y^{\prime} & =B y \\
\phi^{\prime} & =\omega .
\end{aligned}
$$

Summarizing the above we have proved

TheOREM 2.

Suppose

(i) $A=\left[\lambda_{1}, \cdots, \lambda_{n}\right]$ is a real diagonal matrix with

$$
\min _{\alpha \neq \beta}\left|\lambda_{\alpha}-\lambda_{\beta}\right| \geqq r>0 ;
$$

(ii) $P(\phi)$ is an $n \times n$ matrix function of the $m$-vector $\phi$ which is real for real $\phi$, has period $2 \pi$ in each cordinate $\phi_{\alpha}$, and which is holomorphic and satisfies the inequality

$$
|P(\phi)| \leqq M \text { for }|\operatorname{Im} \phi| \leqq \rho \quad(\rho>0) ;
$$

(iii) $\omega$ is a real m-vector such that for all integral $m$-vectors $k \neq 0$

$$
|(k, \omega)| \geqq \gamma|k|^{-\tau} \quad(\tau>m, \gamma>0) .
$$

Then for any $\varepsilon$ such that $0<\varepsilon<\min \left(1, \rho, \frac{1}{2} r\right)$ there exists an (explicitly computable) absolute constant $M_{0}=M_{0}(m, \tau, \gamma, \varepsilon)$ with the property that if $M \leqq M_{0}$ the quasi-periodic linear system 


$$
x^{\prime}=[A+P(\omega t)] x
$$

has a fundamental matrix of the form

$$
X(t)=[I+U(\omega t)] e^{t \theta},
$$

where

(i)' $B=\left[\mu_{1}, \cdots, \mu_{n}\right]$ is a real diagonal matrix with $|B-A| \leqq \varepsilon$ and hence

$$
\min _{\alpha \neq \beta}\left|\mu_{\alpha}-\mu_{\beta}\right| \geqq r-2 \varepsilon>0 ;
$$

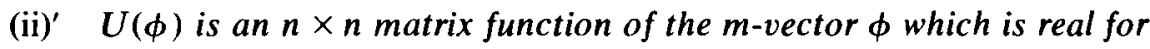
real $\phi$, has period $2 \pi$ in each coordinate $\phi_{\alpha}$, and which is holomorphic and satisfies the inequality

$$
|U(\phi)| \leqq \varepsilon \text { for }|\operatorname{Im} \phi| \leqq \rho-\varepsilon .
$$

The author thanks W. A. Coppel for his kind assistance in the preparation of this paper.

\section{References}

N. N. Bogolyubov, Ju. A. Mitropolśkiī and A. M. Samoilenko (1969), The method of accelerated convergence in non-linear mechanics (Russian) (Izd. "Naukova Dumka", Kiev, 1969).

Alistair Gray (to appear), A reducibility theorem for holomorphic linear systems via an implicit function theorem.

Ju. A. Mitropolskiir and A. M. Samoilenko (1965), 'On constructing solutions of linear differential equations with quasiperiodic coefficients by the method of improved convergence' (Russian), Ukrain, Mat. Z. 17, 42-59.

\section{Economics Department}

La Trobe University

Bundoora Vic., 3083

Australia. 